\title{
Fault Detection through Vibration Signal Analysis based on HSM with TRIPPY Classifier
}

\author{
Sumit Kumar Sar ${ }^{1}$, Ramesh Kumar ${ }^{2}$ \\ ${ }^{1}$ Bhilai institute of Technology \\ Bhilai House, Durg, Chhattisgarh 491001 \\ sumitkumarsar2134@gmail.com \\ ${ }^{2}$ Bhilai institute of Technology \\ Bhilai House, Durg, Chhattisgarh 491001 \\ rameshkumar2332185@gmail.com
}

\begin{abstract}
A proficient fault detection model has to be sketched for detecting slight variations of the vibrating signal of rotating machine whereas the diagnosis process prominently stuck with the inefficient extraction of effectual features of a signal in reduced time. Existence of above stated hilarious issue results in the confinement of inventive Harmonized Swan Machine (HSM) based on the stochastic characteristics of swan, which could collect the RKC (RMS, Kurtosis, Crest factor) signal features for every instantaneous signal unit which eliminates noise thereby reducing pre-processing task which in turn lessens time consumption and at the end yields learned extracted faulty features. Accurate classification of faulty features can be accomplished by casting inimitable Trippy classifier which is designed based on selective predictive character of trippy fish which provokes a good path to provide accurate classification based on learned features. This responsible classifier collectively organises the RKC features of respective signal units and do accurate classification of faulty occurrences based on the features in less time.
\end{abstract}

Keyword: Harmonized Swan Machine, Trippy Classifier, RMS, Crest factor, Kurtosis, feature Extraction

\section{INTRODUCTION}

Rolling bearing of the rotating machine is a significant element and the conditioned rotational machine surveillance prevents the failure of the machine [1]. When faults are generated in the rotating bearing, it causes vibration and noise, which will make the machine breakdown/shutdown [2].In industrial manufacturing of product vacancies, serious vibration of bearings may lead to incorrect operation of the whole scheme[3]. To overcome these issues there are several monitoring ways accessible, that need costly sensors and specialised tools.

This resulted the necessity for a less expensive and precise technique for identifying and preventing the machine faults rather than curing issues. Effective and economical machine failure diagnostics are generally hard owing to big processes such as information acquisition, extraction of the feature, detection of errors and identification structure [4]. In order to extend the life of the bearings, a reliable method is necessary to identify faults in bearings which can reduce the above three measures.

The primary used methods include vibration signal analysis, as these are strongly related to the machine's structural dynamics under surveillance with different fault diagnosis methods[5]. Through adequate process technology the dominant diagnostic data can be obtained through defective vibration signals. Various fault detection methods, for instance the blind source separation [6], wavelet transformation [7-9] and empirical mode decomposition [10-12] for fault detection and diagnosis of mechanical structures have been established and established in latest years with assessment of vibration signals.

These ways type the idea of most applications today, as they provide the chance of time-frequency analysis of signals. Converging need for time frequency analysis, the thought of instantaneous frequency may be determined for each time purpose, making it potential to have a deeper investigation on feature analysis in offset phenomena [13]. It is obtained by Empirical Mode Decomposition (EMD). The vibration signal function of EMD and Fuzzy Entropy and the Back Propagation neural network (BP) were used to diagnose the defects [14].

This notion of neural networks brings together the grouping with the principal component analysis (PCA) of the SelfOrganized Map (SOM) for error diagnosis [15]. The stuck in inefficient feature extraction by PCA brought wavelet entropy along with SVM [16]. Deep learning [17] is one of the strongest representational methods of learning and the Deep Belief Networks (DBNs) [18, 19] pioneers in deep architectural construction.

DBNs could be regarded as an incredibly complex nonlinear extractor of features, in which each hidden layer can display features that obtain greater input correlations.DBNs had previously been used for the recognition of a manually written character [20] and for the recognition of speech[21], for 3D objects [22] and for the extraction of road maps from clustered aerial images [23] and retrieval of knowledge [24].The maintenance department is to inherit the best from above requisites and stay rotating machinery and plant 
equipment in smart operative condition that prevents failure and production loss.

The monitoring of the rotating machine, in particular for industrial engineers, has been hard because the vibration signals of the rotating machines are non-stationary and low energy signals are produced by the robust noise component defects.This necessitate the effective extraction of features for diagnosis which represents main fault information of vibration signals in least time. Though effective extractions of features are made success for each and every instantaneous frequency, there arises a need to predict accurately the fault by precise collection of all the features in less time. Henceforth a proficient algorithm is proposed as said in the proposed methodology.

The remaining of the paper is organized as the related researches in the section 2, proposed methodology in section 3 , results and discussion in section 4, conclusion in section 5 and followed by the references.

\section{LITERATURE SURVEY}

Ince et al. [25] mentioned that early detection of the motor faults is crucial and Artificial Neural Networks (ANNs) were used for feature extraction and classification. Such mounted and handmade features could also be a sub-optimal selection and need a major computational value which stopped their usage for real time applications. This approach was directly applicable to the data (signal) and therefore eliminated the necessity for a separate feature extraction algorithmic rule leading to additional efficient systems in terms of speed and hardware.

Tiwari et al. [ 26] have provided a multi-scale entropy (MPE) and adaptive neuro fuzzy classifier (ANFC) fault designation method. MPE is used to extract features to reduce the vector complexity. For an automatic defect diagnostic procedure, extracted alternatives are entered in the ANFC.This extracted features are provided as input for the intelligent patching matching system named as ANFC. This made use of neural network concept to predict the accurate result.

The very accurate approach to designating defects for lowspeed bearings was stated by Kang et al. [27]. At the beginning of the scheduled approach wavelet-based fault alternatives were obtained which represented different signs of several low speed bearing defects.Every support vector machine was finally taught with its own feature vector and the main discriminatory defects matched the trained outcomes, offering the highest output of classification. The wavelet based scheme provided a high feature contents for satisfying the reliability for classifier.

Myeongsu et al. [28] mentioned an extremely reliable fault designation theme for early low-speed rolling part bearing failures. The 1st method has been calculated by using a rippling package remodel to match incoming emission signal with the multiple relative rippling energy and entropy nodes.A Binary Bat algorithmic rule (BBA) was endorsed by the main fault options square measurement which were filtered from the initially generated function vector by maltreatment discriminative fault assessment.

Shaobo et al. [29] explained that intelligent machine health observance and fault designation were vital for contemporary producing industries. The IDSCNN used deep convolutional neural networks connected with the enhanced theory of Dempster - Shafer based mainly on Gini index evidence fusion.The root-mean square maps of the FFT (Fast Fourier Transformation) are used in convolutional neural networks by two input sensor vibration signals as inputs.

In the aforementioned connected works heap of difficult issues are known for Vibration Signal Analysis. Insulation to supply several range of classification fault sorts in real time observance and additionally process value is high [25]. To tackle mentioned issue [26] is emerged which doesn't scale back the process value and have complexness. To overcome that [27] emerges but it provides average classification accuracy [27]. To improve overall accuracy [28] delineate the fault options square measure, however it lagging to process speed and additionally low performance accuracy achieved. In order to overcome the stated problem, Shaobo gave the IDSCNN a small energy source of weak signals from parts and another big problem is the development of solid noise faults [29]. The above mentioned issues are revised and to unravel this issue an effectual fault diagnosis system is designed is below.

\section{HARMONIZED SWAN MACHINE FOR FEATURE EXTRACTION AND TRIPPY ALGORITHM FOR FAULT DETECTION}

Fault detection is a vital process in analysis of vibrational signal yielded from rotating machinery. In order to extract the featuresuch as RMS, Kurtosis and Crest factor from every signal unit, the vibration signal must be evaluated in detail whereas obtaining features from each individual unit is more tedious. To analyze each nook and corner, here we molded a Harmonized Swan Machine (HSM), a first-hand nature inspired strategy based on the character of Swan bird which has the stochastic nature to accurately extract the needy things based on the characteristic for feature extraction which considers every signal unit. Formally, the effectual features are accurately obtained by the aid of Hilbert Huang Transform which utilizes EMD (Empirical Mode Decomposition) for effective IMF (Intrinsic Mode Function) extraction. The features are learned individually based on the faulty features using DBN which at end yields the learned faulty features. For an efficient fault diagnosis process there is an increased need to exactly classify faulty signals in reduced time which is done with Trippy classifier inspired from the prey catching behavior of Trippy fish. Trippy fish analyses all possibility of prey residence by its knowledge based searching behavior. Similarly, this classifier utilizes the random forest algorithm which makes a list of available outputs but it suffers from accurate prediction. In order to tackle the accurate prediction is offered by the ANFIS which utilizes the knowledge based rule for prediction. Thus the accurate classification of faulty occurrence is done using trippy classifier.The methods used 
are described for each of these steps and the overall proposed architecture described below.

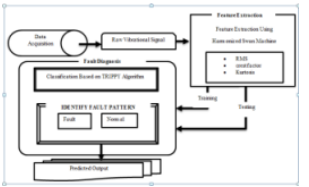

Fig. 1. HSM with Trippy for fault diagnosis

This proposed architecture depicted as fig. 1 showsHarmonized Swan Machine with TRIPPY Algorithm for feature extraction and Fault Diagnosis which aims at improving automatic identification of faults accurately. The system initially starts with the collection of raw data produced by the process of data acquisition from suitable places. The raw vibrated information can be very noisy and also exposed to multiple pollutants.In order to detect features effectively notifying and eradicating noise, Harmonized Swan Machine was developed. This feature extraction technique automatically preprocessed the raw data with the help of high speed training and utilizes the advantage of DBN thereby concentrating on the extraction of only required RKC features. HSM determines the features of the original signal for every instantaneous frequency irrespective of signal type by the greedy layer wise learning enabling fast and active sorts without redundancy and noise. Every signal's RKC features are determined and learnt which holds the faulty signal information. After this process in order to detect the fault diagnosis automatically, an intellectual pattern classification method named trippy classifier is introduced. Finally, fault and normal feature are categorized which is based on the pattern matching concept and the predicted output is finally retrieved.

\subsection{Data Acquisition}

The acquisition of data is the first phase of the fault diagnosis, where machine individualities for further analyzes are evaluated and this article focuses on the diagnosis of vibrated signal errors as discussed in eqn (1).

$$
V_{i}(t)=\left\{V_{1}(t), V_{2}(t) \ldots \ldots . . . V_{m}(t)\right\}(1)
$$

Let $\mathrm{Vi}(\mathrm{t})$ is the set of data of vibrated original signal from other mechanical components of the machine. In rotating machinery,any feature on the raw vibration data can be calculated and the data must be preprocessed early, but in this work the raw data is automatically preprocessed with the help of our proposed feature extraction technique.

\subsection{Harmonized Swan Machine for Feature Selection}

Initially vibrated data are sending to the feature extraction phase for removal of vibrated noise and accurate extraction of proposed features. In order to extract the accuratefeatures we have generated most bang-on tactic HSM which is framed out based on the inspiration of the accurateselective separation of needy elements by a swan bird .Here the needy elements to be the effectual features $\mathrm{RKC}$ and the selective process is done by utilizing DBN inturn for accurate selection HHT is utilized as similar to the stochastic behavioral of swan bird. Every couple of successive levels of the learning process whose joint probability is described as the HSM pre-training procedure is,

$$
J_{h, u}(h, v)=\frac{1}{p(\omega)_{h, v}} \cdot e^{\left(V_{i}(t) w h+v^{T} b+a^{T} h\right)}
$$

The above eqn (1) describes the RBM (Restricted Boltzmann Machine) that has deep learning nature which constituted in each layer $h$ and form the initial DBN structure $p(\omega)_{h, v}$, and then the backward fine tuning is applied to continuous variable v. DBN use the labeled data to train the conditional probability $J_{h \Perp u}(h, u)$ which has the same form as that in DBN layer, and error distributed to fine tuning the network from top to bottom. If the deep-network output unit is compared with the real value, calculate the weight $\mathrm{w}$ error. The error value is returned to receive the error of each layer, and then the efficient weight calculation technique to extract the fault feature is calculated.Now it is necessary to calculate weight with proper bias $b$ to mitigate the training error which is given in

$$
J_{h, u}(h, v)=\frac{1}{p(\omega)_{h, u}} \cdot e^{\left(V(t) w h+(v-b)^{T}(v-b)+a^{T} h\right)}(3)
$$

By maximizing the probability of joint probability RBM parameters are trained effectively, without supervisionusingequation (2)

$$
L=\Pi_{t} \sum_{h} J_{h, u}(h, v(t))(4)
$$

This $L$ is over training samples of vibrated signal $v(t)$.To extract IMFs in feature extraction from a complicated signal, thetraining phase of HSM algorithm adopt the EMD technique to determine the monocomponent of the original vibrated raw signal.

EMD can disintegrate a signal into an IMF (Intrinsic Mode Function), a collection of single-component signals. An IMF satisfies two requirements (1) in the total set of data; the extreme number and the number of zero crossings must be equal to, or at most different by, one; and (2) at any point: the mean value of the envelope defined by the local maximum and the local minima defined envelope must be nil.

By obtaining the instaneous monocomponent signal, IMF is obtained in order to analyse the signal individually for better confinement of effectual features from each unit which is obtained by EMD methodology. A signal $V$ (t) may be displayed for eqn (2) following EMD as in eqn 4,

$$
V(t)=\sum_{i=1}^{n} x_{i}+y_{n}(5)
$$

Where $x_{i}$ is the ith decomposed IMF of the $\operatorname{signal}_{V(t)}, y_{n}$ is the noisy signal. Here the EMD determines the monocomponent of the original signal individually but there is an extended need to analyze every mono-component individually based on its instantaneous frequency, so that the effectual features can be obtained from each individual of vibrated signal and also in addition this EMD processes results with some limited amount of noise, which is also to be tackled. Above stated issues are tackled by taking the Hilbert Huang 
transform which effects a selective preprocessing of the effectual signal features obtained. HHD is therefore used on the two sides of Eq. (5), the following equation (6) may be performed: the Hilbert Huang spectrum $V(t), P(\omega, t)$ :

$$
P(\omega, t)=\operatorname{Re} \sum_{i=1}^{n} v_{i}(t) e^{j \int \omega_{i}(t) d t}(6)
$$

Where $\operatorname{Re}$ is the operator of real part, $v_{i}(t)$ and $\omega_{i}(t)$ denote the functions of the amplitude and instantaneous frequency, respectively. Note that the residual term $y_{n}$ in Eq. (5), which occupies very little energy of the signal, is ignored that is the noise is avoided which is equivalent to preprocessing of signal by utilizing the HSM nature based on eqn (6) , which in turn reduces the time factor for extraction of faulty features.

The Hilbert - Huang marginal spectrum transforms which RKC extract features can be characterized in relation to moment by an integrated spectrum as in eqn (7)

$$
p(\omega)=\int_{0}^{T} P\left(\omega, t_{(\text {RMS }, \text { Crest Factor, Kurtosis })}\right) d t
$$

Where $\mathrm{T}$ is no of the features of $V(t)$ and $P(\omega, t)$ can exactly describe the extraction of effectual faulty feature such as RMS, Kurtosis, and Crest of every individual IMF monocomponent with defined time interval. The advantage of our extraction technique with the help of HHT is to obtain all feature extracted within instantaneous frequency limit without noise existence.

Such that calculating IMF and Residue using HSM, the machine noises are eradicated and raw data are conditioned automatically with the help of HSM Training. Thus on the whole feature Extraction by Harmonized Swan Machine (HSM) is the process of precisely extracting required features for the faulty signal from the raw vibrated signal. The overall architecture of Harmonized Swan Machine is described below fig 2 .

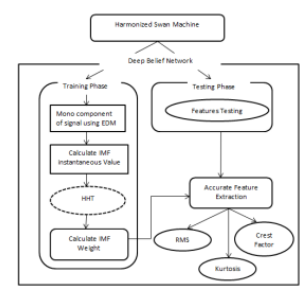

Fig. 2. Harmonized Swan Machine schematic architecture

Normally, the factors such as RMS, Kurtosis, and Crest factor are seen to be prominently projected criteria of a faulty signal when compared with normal vibrated signals. Therefore, by analyzing the RKC features it is easier for knocking out of the faulty signals without random searching which is the reason for deeply learning the three parameter RKC. This RKC interprets as the key to judge the faulty signals occurred. The description of RKC features is given below,

(i) $R M S$
A time analysis feature, which is the measurement of the power content of the vibration signature, is the Root Mean Square (RMS). This feature is good for tracking the total noise level, but it doesn't give data about the missing element.The detection of a significant gap in rotating systems can be highly efficient. In this equation is the root average square value, $\mathrm{u}(\mathrm{t}) \mathrm{n}$ over $\mathrm{N}$ length, of a trained data series.

$$
R M S=\sqrt{\frac{1}{N}} \times \sum_{n=1}^{n} v(t)_{n}^{2}
$$

\section{(ii) Kurtosis}

Kurtosis is described as the distribution and measures a distribution's relative peak or flatness compared to a normal distribution. Kurtosis measures the size of the distribution tails and is used in a collection of information as a measure of the significant peaks.Due to the enhanced vibration rate of a gear wear and break, this function should indicate a mistake. The kurtosis equation is determined by:

$$
\text { Kurtosis }=\frac{\sum_{n=1}^{N}[v(t)-\mu]^{4}}{N \times\left(\sigma^{2}\right)^{2}}
$$

Where $\mathrm{v}(\mathrm{t})$ is the raw time series at point $\mathrm{n}, \mu$ is the average of the data that is trained; $\sigma 2$ is the variance and $\mathrm{N}$ the total data points.

\section{(iii) Crest Factor}

The "crest factor," the ratio of the input signal peak to the RMS stage, is a better metric. The peaks of the time series signal therefore improve the importance of the crest factor. The crest factor equation is given below:

$$
\text { Crestfactor }=\frac{\text { Peak Level }}{R M S}(10)
$$

Finally features are extracted perfectly without noise and redundancy. The main advantage of our proposed feature extraction technique is the adaptability of all signal kind due to the joint probability distribution parameter of RBM.After acquisition of features, the group of all those extracted features must automatically complete a smart pattern classification to fulfill the defective diagnosis.In this work TRIPPYAlgorithm proposed to fulfil the fault diagnosis automatically by grouping all those extracted monochrome features which is achieved by the predictable ensemble nature of the classifierand is proposed below.

\subsection{Fault Diagnosis Using Trippy Algorithm}

The knock out classification is carried out in our work by utilizing most pioneering nature inspired classifier baptized as trippy classifier based on the deep searching and accurate predictive nature of trippy fish whereas this classifier offers a deep learning or searching by means of the randomized character of RF (Random forest) also predictive function by means of ANFIS with knowledge based prior judging. Thus once the learned faulty features are feeded to this classifier which analysis deeply and offers accurate diagnosis which inturn separates the classified faulty signals.

Initially the output results $\mathrm{Li}$ (1) and $\mathrm{Li}$ (2) of the trainedHSM should be fed into the trippy classifier which 
offers deep learning by means of RF. The data fusion training of the faulty features is defined as

$$
\begin{aligned}
& \mid \begin{array}{l}
i \text { (1) } \\
\arg _{i} \max \\
i
\end{array} \\
& {\left[\begin{array}{ll}
i(2) & \operatorname{arq}_{i} \max L_{i}^{(2)}=\operatorname{arq}_{i} \max p\left(\omega_{i}\right)
\end{array}\right]_{\text {Wherei }}=1,2,3 \ldots, \mathrm{m}} \\
& \text { where }\left\lfloor p\left(\omega_{i}\right)=p\left(\omega_{1}\right)+p\left(\omega_{2}\right)+\ldots . .+p\left(\omega_{m}\right)\right\rfloor
\end{aligned}
$$

The Learning Features $\operatorname{Li}\left(p\left(\omega_{i}\right)\right)$ is imported from eqn 6 that consist of RKC, which is utilized for diagnosing the fault.

$$
L_{i}=\left[L_{1}^{(1)}, L_{2}^{(2)}, \ldots, L_{m}^{k} \mid(13)\right.
$$

The above eqn (12) is used to make a tree using a tree classification algorithm, using a distinct bootstrap sample from the initial information. Where ' $\mathrm{m}$ ' is the number of featureswhich are extracted from HSM. Once the forest has been established, objects to be trained are placed in the forest for practice under each of the trees. The training features are described in the eqn (13)

$$
\omega_{i}=L_{i} \max p\left(\omega_{i}\right)(14)
$$

Followingly, accurate prediction process of the trippy classifier after deep learning is done by utilizing theNeuro fuzzy interference system which utilizes knowledge based pattern identification for the prediction of vibrated fault signal.

ANFIS modeling more systematic and less reliant on expert knowledge, thus creating more objective. Without loss of generality and for simplicity, it is assumed that the ANFIS under consideration has two inputs $x$ and $y$, and one output $f$. Assume that the rule base only includes two Sugeno rules of first order sort; a straightforward instance with the rule base is the following to explain the specified notion of ANFIS construct:

$$
\text { Rule } 1 \text { If } x \text { is } A 1 \text { and } y \text { is } B 1 \text {, then } \mathrm{f} 1=\mathrm{p} 1 \mathrm{x}+\mathrm{q} 1 \mathrm{y}+\mathrm{r} 1 \text {, }
$$

Rule 2 If $x$ is $A 2$ and $y$ is $B 2$, then $f 2=p 2 x+q 2 y+r 2$, (16)

where $\mathrm{x}$ and $\mathrm{y}$ are the inputs, $\mathrm{A} 1 ; \mathrm{A} 2$; $\mathrm{B} 1$ and $\mathrm{B} 2$ are fuzzy sets which represents the range of the design parameters $\mathrm{p} 1$; $\mathrm{q} 1 ; \mathrm{r} 1 ; \mathrm{p} 2 ; \mathrm{q} 2$ \& $\mathrm{r} 2$ determined during trainee procedures are design parameters and associated Trippy equivalent architecture is displayed in Fig 3

$$
\text { overall output }=\sum \omega_{i} f_{i}=\frac{\sum_{i} \omega_{i} f_{i}}{\sum_{i} \omega_{i}}
$$

This algorithm consists of two steps and is a mixture of the gradient descent strategy and the least quadratic estimation. The first phase involves fixing the premise parameters and identifying the appropriate consecutive parameters with the least estimates of squares.In the second phase, however, the following parameters will have to be fixed and the premise parameters will be update based on the error signals by the back propagation gradient descent technique thus yielding the optimized classification by pointing the correct prediction. Finally the precise diagnosis of the behavior of rotating machine faults is identified in fast prediction time without noise redundancy.

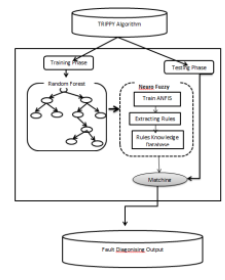

Fig. 3.Trippy Classifier for fault Diagnosing

Thus by the usage of efficient feature extraction by utilizing HSM and the intelligent pattern recognition by means of trippy classifier correctly classifies fault with the assistance of learning based feature extraction. This is the well-lit processdue to the expellation of preprocessing and accurate prediction process which eliminates the esteemed usage of time. The result validation in the below section will be an added proof for the efficiency of the work.

\section{RESULTS AND DISCUSSION}

In section 3 above, the suggested method is outlined and the detailed explanation is provided and its performance is analyzed. The suggested technique is introduced with the following system specifications in the MATLAB working platform.

$\begin{array}{ll}\text { Platform } & \text { : MATLAB 2015a } \\ \text { OS } & \text { : Windows } 8 \\ \text { Processor } & : \text { Intel core i5 } \\ \text { RAM } & : 8 \text { GB RAM }\end{array}$

\subsection{Dataset Collection:}

Dataset was gathered at the Department of Electrical Engineering at a single point of air compressor reciprocation. The air compressor specification is as follows:

- Air Pressure Range: 0-500 lb/m2, 0-35 Kg/cm2

- Induction Motor: 5HP, 415V, 5Am, $50 \mathrm{~Hz}$, 1440rpm

- $\quad$ Pressure Switch: Type PR-15, Range 100-213 PSI

The data sets include several states including the good condition of the piston, 7 defective countries, the fault of the Leakage Inlet Valve (LIV), Leakage Outlet Valve (LOV), the non-Reversal Valve fault (NRV), Piston ring fault, Flywheel fault, Rider belt fault, and Bearing fault. Faults have been placed in the air compressor to collect recordings from all these states.

\subsection{Simulation result}

The vibrated bearing fault raw dataset are plotted in the given below fig 4and the histogram figures are plotted in the fig 5 .

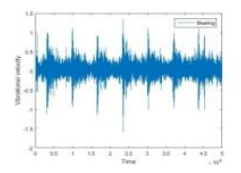

Fig. 4. original raw bearing vibrated signal of bearing 

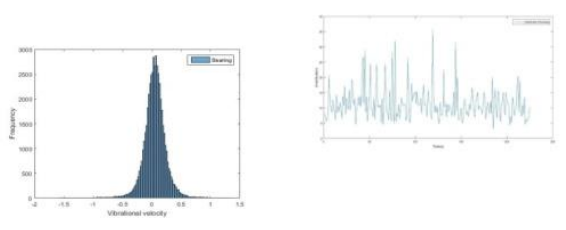

Fig. 5. Histogram of raw vibrated signal of bearing

The vibrated signal is focusing on the feature extraction like RMS, kurtosis, Crest factor. In this Paper Harmonized Swan Machine is utilized for accurate extraction of feature. EMD determines the monocomponent of original signal Our proposed intrinsic mode function of the original signal is described in fig 6,7 and 8 .

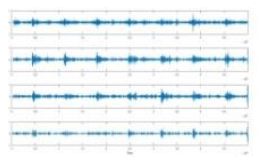

Fig. 6. IMF 1 values of original signal

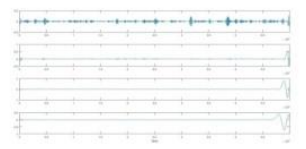

Fig. 7. IMF 2 values of original signal

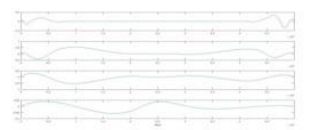

Fig. 8. IMF 3 values of original signal

After EMD technique the instantaneous frequency is calculated using HilbertHuang Transform. In this below figure 9 shows that the spectrogram of HHT of instantaneous frequency signal.

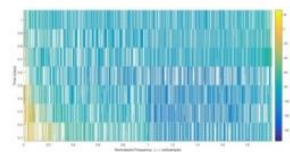

Fig. 9. EMD based Hilbert-Huang transform

After that process some faulty signal are available in the above information. In order to avoid this issue HSM is utilized for deep learning. The learning behavior of HSM is used to extract the needed feature for fault diagnosis in precise way with the help of IMF calculation based on the nature of HSMwhich is widely used monochrome of individual frequency signal. Finally precise noise features are eliminated and our needed features such as RKS are accurately extracted within the less time. After this process our proposed feature extraction are describedin the below fig 10,11,12.
Fig. 10. Crest factor of bearing

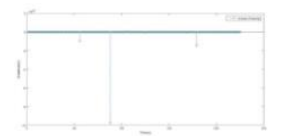

Fig. 11. Kurtosis of bearing

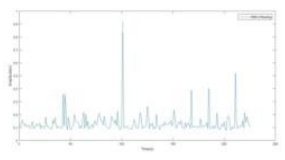

Fig. 12. RMS of bearing

In order to predict the accurate vibrated signal, the intelligent based pattern classification is needed, so in this research paper focus on TRIPPY for predict the accurate faulty signal automatically. In the given below fig 13 describes the decision tree but it doesn't predict the accurate signal because it has apriori knowledge less nature.

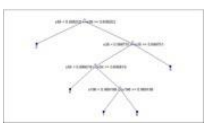

Fig. 13. Random forest decisionTree

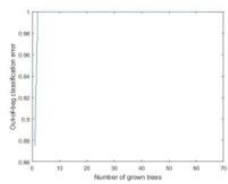

Fig. 14. Error of prediction in RF

After that in order to predict the accurate vibrated signal neuro fuzzy enzymes are proposed to classify the accurate vibrated faulty signal in the given below fig 15: describes the decision tree of after Random forest prediction using proposed randomized neuro fuzzy enzyme.

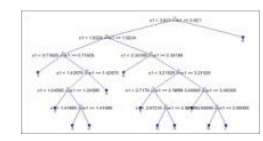

Fig.15. Trippy algorithm decision Tree

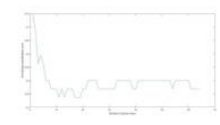


Figure 16:proposed accurate faulty signal detection basd on decision Tree

In the above fig 16 describes the faulty signal detection in the rotation machine using TRIPPY Classifier.Finally the accurate behaviour of faulty state are identified in best manner. Our proposed algorithm has knowledge based rule automatic detection is used to diagonsing the faulty signal.

\subsection{Comparison Analysis}

Comparison was made by proper analysis of computation time, accuracy, DiagnosisAccuracy, Testing Prediction Time and Diagonosis Noise are described below section.

\subsubsection{Computation time:}

Computation time during feature extraction is defined as the time required for extracting the necessary features from raw vibrated data.The computation formulas are described below.

Computation Time $=$ Starting Time of featureextraction - Ending Time of featureextraction

TABLE 1:

computation Time of proposed transform and existing Transform

\begin{tabular}{|l|l|c|}
\hline \multicolumn{1}{|c|}{ Sl.no } & \multicolumn{1}{|c|}{ Algorithm } & Run Time \\
\hline 1 & TDSP & 0.034 \\
\hline 2 & Fast flourier Transform & 0.013 \\
\hline 3 & $\begin{array}{l}\text { Morlet wave let } \\
\text { Transform }\end{array}$ & 0.072 \\
\hline 4 & $\begin{array}{l}\text { Discrete wave let } \\
\text { transform }\end{array}$ & 0.092 \\
\hline 5 & $\begin{array}{l}\text { Short time Fourier } \\
\text { transform }\end{array}$ & 0.648 \\
\hline 6 & Wigner wile Distribution & 0.008 \\
\hline 7 & $\begin{array}{l}\text { Pseudo- Wigner wille } \\
\text { Distribution }\end{array}$ & 2.120 \\
\hline 8 & Auto correlation & 0.028 \\
\hline 9 & $\begin{array}{l}\text { Updated more let } \\
\text { Transform }\end{array}$ & 0.029 \\
\hline 10 & Convolution with sine & 0.012 \\
\hline 11 & S-transform & 5.328 \\
\hline $\mathbf{1 2}$ & Proposed & $\mathbf{0 . 0 2 1}$ \\
\hline
\end{tabular}

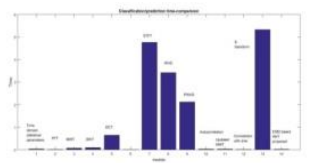

Figure 17: comparison of proposed and existing transform for feature extraction

In figure 17, each transform is presented with its respective features, and the average calculation time per record. The calculation times over 1000 records showed these values, which describes the computation time for existing transform for analyzing the selected features, those using FFT, WPT, DCT, WVD, STFT the vales are described in table 1 and our proposed feature extraction which utilize training with EDM based HHT attain the lower computation time when compared to existing works.

\subsubsection{Accuracy:}

The accuracy of a test is its capacity to properly distinguish between the deficient and healthy situation. In all assessed cases, the proportion of genuine positive and real negative should be calculated in order to assess the accuracy of a test. This can be indicated mathematically as:

$$
\text { Accuracy }=\frac{a+d}{a+d+c+b}
$$

Where, True positive $(\mathrm{a})=$ the number of features correctly identified as faults,

False positive (c) $=$ the number of features incorrectly identified as faults,

True negative $(d)=$ the number of features correctly identified as Normal,

False negative $(b)=$ the number of features incorrectly identified as Normal.

TABLE 2:

Accuracy comparison of various bearing and proposed bearing

\begin{tabular}{|c|c|c|}
\hline Sl.no & Algorithm & Total (sec) \\
\hline 1 & KNN & 86.67 \\
\hline 2 & PNN & 90 \\
\hline 3 & RBN & 96.67 \\
\hline 4 & PSO-SVM & 96.67 \\
\hline 5 & PROPOSED & 98.2 \\
\hline
\end{tabular}

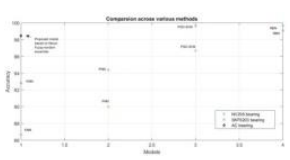

Fig.18. Accuracy comparison for various bearing method of existing and proposed

In the above fig 18 offerings the Accuracy prediction time per recording for computing each transform and its corresponding fault diagnosis, which describes the accuracy forexisting andanalyzing the diagnosing the fault, those using KNN, PNN, RBN, PSO-SVM, and proposed the values are described in table 2 and our proposed Trippy algorithm, which attain the higher accuracy when compared to existing algorithm.

\subsubsection{Testing prediction Time:}

The testing prediction time is defined as the time taken to predict the precise fault.

TABLE 3:

overall prediction using existing and proposed

\begin{tabular}{|l|l|l|}
\hline Sl.no & Algorithm & $\begin{array}{l}\text { Total } \\
\text { (sec) }\end{array}$ \\
\hline 1 & KNN & 0.51 \\
\hline 2 & PNN & 0.064 \\
\hline 3 & RBN & 20.6307 \\
\hline 4 & PSO-SVM & 0.05033 \\
\hline 5 & PROPOSED & 0.068 \\
\hline
\end{tabular}




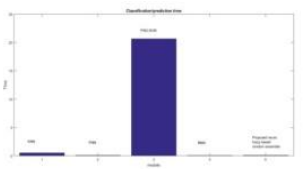

Fig. 19. overall prediction using existing and proposed

In the above fig 19 that describe the overall prediction comparison of our proposed algorithm and existing algorithm such as KNN, PNN, RBN, PSO-SVM and proposed values are plotted in the table:3.Finally our proposed Trippy classifier achieve less prediction time when compared to all other existing algorithm and also precisely diagnose the fault with 0.068 secs which is quite higher. This time is acceptable since we do training twice and we are receiving this less time.

\subsubsection{Comparison of Diagnosis Accuracy:}

The Diagnosis Accuracy is defined as the overall probability that a fault will be correctly classified based on the learning sample data set. The Diagnosis Accuracy formula is described below

$$
\text { Diagnosis Accuracy }=\frac{a+d}{a+b+d+c}
$$

Table 4:Comparison of Diagonsis Accuracy Proposed and Exiting Method

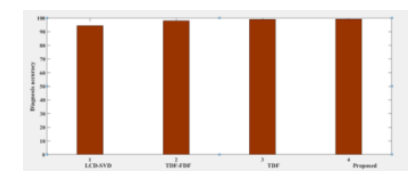

Fig. 20. Diagnosis Accuracy of different and our proposed Method

In the above fig 20, that describes the comparison of different existing feature selection method and our proposed for fault diagnosis of rotating machine. In existing work large data set are used to extracting the selected features for diagnosing the fault. Similarity, in this work we are focusing to learned the large number of vibrated raw signal for needed features selection.In table 5, that describes the training and testing samples for accurate feature selection using different classes for featuresextraction. Overallperspective our proposed feature selection technique attain the higher accuracy for selecting the feature because the training data set are huge compared to existing works such as LCD-SVD, TDF-FDF and TDFwhich is easily attainable to extract the feature. But our proposed feature such RMS,Crest factor and Kurtosis, which are not easily achievable to extract the feature but in this work our proposed algorithm to extract the accurate needed feature easily with the help of stochasticnature. Finally our proposed diagnosing accuracy value is being increased 98.28 when compared to all other existing classifier like CRO,PNN, and Random forest.

\subsubsection{Comparison of diagnosis noise:}

The noise diagnosis is described with the MSE (Mean Squared Error) most readily. Due to the monochrome noise free characteristic I and its noisy approximation K, MSE is described as:

$$
M S E=\frac{1}{m n} \sum_{i=0}^{m-1} \sum_{j=0}^{n-1}[I(i, j)-k(i, j)]^{2}
$$

PSNR (in $\mathrm{dB}$ ) is defined as,

$$
P S N R=20 . \log _{10}\left(M A X_{I}\right)-10 \cdot \log _{10}(M S E)
$$

Here, MAXI is the extremeprobableerror esteem of the vibrated signal. If the faults are shown with a sample of 8 bits,

\begin{tabular}{|c|c|c|c|c|c|c|c|c|c|c|}
\hline \multirow{5}{*}{\multicolumn{2}{|c|}{$\begin{array}{c}\text { TABLE 5: } \\
\text { Compariso } \\
n \text { of } \\
\text { diagnosis } \\
\text { results } \\
\text { under } \\
\text { different } \\
\text { noise } \\
\text { environme } \\
\text { nt }\end{array}$}} & $\begin{array}{l}\text { Si. } \\
\text { No }\end{array}$ & $\begin{array}{c}\text { Feat } \\
\text { ures } \\
\text { Algo } \\
\text { rith } \\
\text { m }\end{array}$ & \multicolumn{2}{|c|}{$\begin{array}{c}\text { Classif } \\
\text { ier } \\
\text { Algorit } \\
\text { hm }\end{array}$} & $\begin{array}{c}\text { No.of } \\
\text { trainin } \\
\text { g } \\
\text { sampes }\end{array}$ & \multicolumn{2}{|c|}{$\begin{array}{c}\text { No.of } \\
\text { testing } \\
\text { sample } \\
\text { S }\end{array}$} & $\begin{array}{c}\text { No. } \\
\text { of } \\
\text { class } \\
\text { es }\end{array}$ & $\begin{array}{c}\text { Diag } \\
\text { nosi } \\
\text { s } \\
\text { accu } \\
\text { racy }\end{array}$ \\
\hline & & 1 & $\begin{array}{c}\text { LCD } \\
- \\
\text { SVD }\end{array}$ & \multicolumn{2}{|c|}{$\begin{array}{l}\text { CRO- } \\
\text { SVM }\end{array}$} & 240 & \multicolumn{2}{|c|}{80} & 4 & 100 \\
\hline & & 2 & $\begin{array}{c}\text { TDF } \\
- \\
\text { FDF }\end{array}$ & \multicolumn{2}{|c|}{ PNN } & 240 & \multicolumn{2}{|c|}{80} & 4 & $\begin{array}{c}94.3 \\
8\end{array}$ \\
\hline & & 3 & TDF & \multicolumn{2}{|c|}{$\begin{array}{l}\text { Rando } \\
\mathrm{m} \\
\text { forest }\end{array}$} & 200 & \multicolumn{2}{|c|}{200} & 4 & $\begin{array}{c}98.0 \\
4\end{array}$ \\
\hline & & 4 & $\begin{array}{c}\text { HS } \\
\text { M }\end{array}$ & \multicolumn{2}{|c|}{ Trippy } & 225 & \multicolumn{2}{|c|}{50} & 8 & $\begin{array}{c}98.2 \\
8\end{array}$ \\
\hline $\begin{array}{l}\text { Diagn } \\
\text { osis } \\
\text { Classi } \\
\text { fier }\end{array}$ & $\begin{array}{l}12 \mathrm{~d} \\
B\end{array}$ & $\begin{array}{l}13 \\
d B\end{array}$ & $\begin{array}{l}14 \\
d B\end{array}$ & $\begin{array}{l}15 \\
d B\end{array}$ & $\begin{array}{l}16 \\
d B\end{array}$ & $\begin{array}{l}18 \\
d B\end{array}$ & $\begin{array}{l}20 \\
d B\end{array}$ & $\begin{array}{l}22 \\
d B\end{array}$ & $\begin{array}{l}24 \\
d B\end{array}$ & $\begin{array}{l}26 \\
d B\end{array}$ \\
\hline $\begin{array}{l}\text { Rando } \\
\mathrm{m} \\
\text { Forest }\end{array}$ & $\begin{array}{l}74 . \\
78\end{array}$ & $\begin{array}{l}85 . \\
82\end{array}$ & $\begin{array}{l}92 . \\
95\end{array}$ & $\begin{array}{l}95 . \\
34\end{array}$ & $\begin{array}{l}97 . \\
74\end{array}$ & $\begin{array}{l}98 . \\
60\end{array}$ & $\begin{array}{l}99 . \\
00\end{array}$ & $\begin{array}{l}99 . \\
26\end{array}$ & $\begin{array}{l}99 . \\
46\end{array}$ & $\begin{array}{l}99 . \\
53\end{array}$ \\
\hline ELM & $\begin{array}{l}65 . \\
53\end{array}$ & $\begin{array}{l}69 . \\
53\end{array}$ & $\begin{array}{l}73 . \\
01\end{array}$ & $\begin{array}{l}76 . \\
39\end{array}$ & $\begin{array}{l}79 . \\
72\end{array}$ & $\begin{array}{l}85 . \\
48\end{array}$ & $\begin{array}{l}91 . \\
40\end{array}$ & $\begin{array}{l}96 . \\
81\end{array}$ & $\begin{array}{l}98 . \\
83\end{array}$ & $\begin{array}{l}99 . \\
37\end{array}$ \\
\hline PNN & $\begin{array}{l}63 . \\
25\end{array}$ & $\begin{array}{l}71 . \\
64\end{array}$ & $\begin{array}{l}79 . \\
16\end{array}$ & $\begin{array}{l}84 . \\
84\end{array}$ & $\begin{array}{l}88 . \\
77\end{array}$ & $\begin{array}{l}93 . \\
43\end{array}$ & $\begin{array}{l}96 . \\
51\end{array}$ & $\begin{array}{l}97 . \\
81\end{array}$ & $\begin{array}{l}98 . \\
28\end{array}$ & $\begin{array}{l}98 . \\
87\end{array}$ \\
\hline SVM & $\begin{array}{l}66 . \\
87 \\
\end{array}$ & $\begin{array}{l}73 . \\
32 \\
\end{array}$ & $\begin{array}{l}78 . \\
85\end{array}$ & $\begin{array}{l}83 . \\
59\end{array}$ & $\begin{array}{l}87 . \\
66\end{array}$ & $\begin{array}{l}92 . \\
28\end{array}$ & $\begin{array}{l}95 . \\
68\end{array}$ & $\begin{array}{l}97 . \\
24\end{array}$ & $\begin{array}{l}98 . \\
11\end{array}$ & $\begin{array}{l}98 . \\
67\end{array}$ \\
\hline $\begin{array}{l}\text { Propo } \\
\text { sed }\end{array}$ & $\begin{array}{l}72 . \\
98\end{array}$ & $\begin{array}{l}76 . \\
28\end{array}$ & $\begin{array}{l}80 . \\
95\end{array}$ & $\begin{array}{l}84 . \\
64\end{array}$ & $\begin{array}{l}87 . \\
56\end{array}$ & $\begin{array}{l}3 . \\
51\end{array}$ & $\begin{array}{l}97 . \\
89\end{array}$ & $\begin{array}{l}98 . \\
28\end{array}$ & $\begin{array}{l}99 . \\
68\end{array}$ & $\begin{array}{l}99 . \\
73\end{array}$ \\
\hline
\end{tabular}
it's 255. More usually, samples with linear PCM with B bits per sample are depicted.

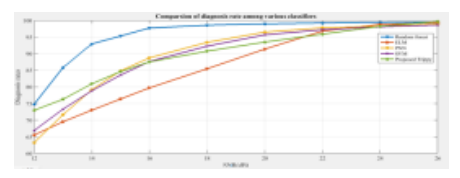


Fig. 21.Diagnostic results of employed classifiers with different SNRs.

In figure 21 above it can be evident that when Trippy Classifier handles the sample samples with various noise concentrations, it achieves statistically significant gains over comparison techniques. As shown by the values in Table 4, a clear downward trend will be shown for ELM, PNN, SVM and Trippy when the SNR is below $22 \mathrm{~dB}$, while Trippy is still significantly good at the SNR due to our strong noiseresistance algorithm.

4.2.6. Time consumption of prediction in different classifiers:

Time consumption of prediction is the ratio of total time taken for prediction to the time taken for completion.

$$
\text { Time Consumption }=\frac{\text { Time taken to predict the features }}{\text { Total time taken for the completion of the process }}
$$

TABLE 6:

Time consumption for prediction in different classifiers

\begin{tabular}{|l|l|l|}
\hline Sl.no & Algorithm & $\begin{array}{l}\text { Prediction } \\
\text { Time }\end{array}$ \\
\hline $\mathbf{1}$ & Random forest & $5.08 \mathrm{~ms}$ \\
\hline $\mathbf{2}$ & ELM & $0.60 \mathrm{~ms}$ \\
\hline $\mathbf{3}$ & PNN & $8.44 \mathrm{~ms}$ \\
\hline $\mathbf{4}$ & SVM & $0.50 \mathrm{~ms}$ \\
\hline $\mathbf{5}$ & Proposed & $\mathbf{0 . 4 9} \mathrm{ms}$ \\
\hline
\end{tabular}

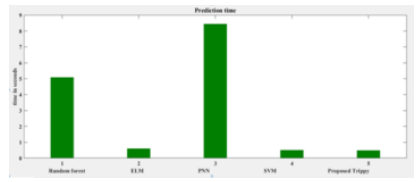

Fig. 22.Time consumption of prediction in different classifiers

In the above fig:22 that describes the overall diagonising prediction time compared with existing work and our proposed Trippy Classifier achieve the less prediction time using large data set for training and testing. The advantage of our proposed classifier to fulfill in least time prediction in fault diagnosing in the rotating machinery.In table 6 values indicated the prediction time comparision of different method such as Random Forest achieves the prediction value $5.08 \mathrm{~ms}$,ELM method attain $0.60 \mathrm{~ms}$,PNN prediction time consumption value is $8.44 \mathrm{~ms}$, the prediction of fault diagnosing time SVM method is $0.50 \mathrm{~ms}$ and our proposed method HSM with Trippy Algorithm achieves the less prediction time due to the selective predictive nature of trippy classifier, which attain $0.49 \mathrm{~ms}$ prediction time when compared to existing our proposed approach has attain the better prediction time for fault diagonosis.Consequently, our proposed algorithm automatically detects the fault in the rotating vibrated machine in early stage and also saves sector from severe machine failure losses.

\section{CONCLUSION}

This work creates an approach to rotating machinery fault diagnostics by utilizing HSM based Trippy classifier. HSM analyses the accurate effective fault features whereas the trippy classifier predicts fault occurrence selectively by means of extracted faulty features. The aforementioned strategies are formally enforced with great precision on the air compressor and can detect identified defects. In reality, vibrational signals were analyzed to detect errors with computation time of 0.021 secs, $98.2 \%$ accuracy and prediction time of $0.049 \mathrm{~ms}$ using our structured feature extraction and classification framework.

\section{REFERENCES}

[1] Dou, Dongyang, and Shishuai Zhou."Comparison of four direct classification methods for intelligent fault diagnosis of rotating machinery".Applied Soft Computing,vol.46,pp.459-468, 2011

[2] P.K. Kankar , C .Satish Sharma , S.P.Harsha ."Fault diagnosis of ball bearings using machine learning methods". Expert Syst. Appl., vol.38, pp.1876-1886,2011.

[3] Riaz, Saleem, Hassan Elahi, KashifJavaid, and TufailShahzad."Vibration feature extraction and analysis for fault diagnosis of rotating machinery-A literature survey."Asia Pacific Journal of Multidisciplinary Research, 5(1), 103-110,2017.

[4] Zhu, Keheng, Xigeng Song, and DongxinXue."A roller bearing fault diagnosis method based on hierarchical entropy and support vector machine with particle swarm optimization algorithm.Measurement,"vol. 47, pp.669675,2014

[5] F .Al-Badour , M. Sunar , L.Cheded . "Vibration analysis of rotating machineryusing time-frequency analysis and wavelet techniques.Mech". Syst. Signal Process,vol.25,no.6,pp. 2083-2101,2011.

[6] Z .Li , X .Yan , Z. Tian ,et al. "Blind vibration component separation and nonlinear feature extraction applied to the non-stationary vibration signals for the gearbox multifault diagnosis.Measurement", vol.46,no.1, pp.259-271, 2013.

[7] W .He, Y .Zi, B .Chen,et al. "Automatic fault feature extraction of mechanical anomaly on induction motor bearing using ensemble super-wavelet transform.Mech. Syst". Signal Process, vol. 54,no.55, pp.457-480,2015.

[8] N .Saravanan , K.I. Ramachandran ..."Incipient gear box fault diagnosis using discrete wavelet transform (DWT) for feature extraction and classification using artificial neural network (ANN).Expert Syst. Appl"., vol.37,no.6, pp.4168-4181,2010.

[9] Q .Hu , Z .He, Z .Zhang, Y. Zi. "Fault diagnosis of rotating machinery based on improved wavelet package transform and SVMs ensemble. Mech." Syst. Signal Process,vol.21,no.2,pp. 688-705,2007.

[10] Y .Lei , J. Lin , Z .He , et al. "A review on empirical mode decomposition in fault diagnosis of rotating machinery.Mech". Syst. Signal Process, vol.35,no.1-2, pp.108-126,2013. 
[11] Q .Gao , C .Duan , H .Fan, et al. "Rotating machine fault diagnosis using empirical mode decomposition.Mech. Syst." Signal Process,vol.22,no.5,pp.1072-1081, 2007.

[12] Y .Li, M .Xu, Yu Wei, et al. "An improvement EMD method based on the optimized rational Hermite interpolation approach and its application to gear fault diagnosis.Measurement, "vol.63, pp.330-345,2015.

[13] Kountchev, Roumen, and Kazumi Nakamatsu. "New Approaches in Intelligent Image Analysis, Springer." 2016.

[14] J.J. Zhao , Y .Yang, et al. (2013)."Application of Empirical Mode Decomposition and Fuzzy Entropy to HST Fault Diagnosis".The $8^{\text {th }}$ International Conference on Intelligent Systems and Knowledge engineering.

[15] Z.C. Li . "A Simple SOM Neural Network Based Fault Detection Model for Fault Diagnosis of Rolling Bearings". Applied Mechanics and Materials,vol.397, pp.1321-1325,2013.

[16] N .Qin , W.D. Jin, J. Huang, et al."HST Bogie Fault Signal Analysis Based on Wavelet Entropy Feature.Advanced Materials Research",vol.753, pp.2286$2289,2013$.

[17] X.L. Zhang. "Learning deep representation without parameter inference for nonlinear dimensionality reduction." arXiv preprint arXiv,pp. 1308.4922,2013.

[18] G.E. Hinton , R.R Salakhutdinov . "Reducing the dimensionality of data with neural networks.Science",vol.313, pp.504-507,2006.

[19] G.E.S .Hinton, Y.W. Osindero ."The, A fast learning algorithm for deep belief nets, Neural computation 18" pp.1527-1554,2006.

[20] H .Lee, R .Grosse , R. Ranganath, et al. "Convolutional deep belief networks for scalable unsupervised learning of hierarchical representations.Proceedings of the 26th Annual International Conference on Machine Learning,"pp.609-616,2009.

[21] A .Mohamed , G.E .Dahl, , G .Hinton. "Acoustic modeling using deep belief networks."IEEE Transactions on Audio, Speech, and Language Processing, vol.20, pp.14-22,2012.

[22] V. Nair , et al."3-d object recognition with deep belief nets".Advances in Neural Information Processing Systems, vol.22, pp.1339-1347,2009.

[23] V .Mnih, et al." learning to detect roads in high-resolution aerial images."Computer Vision - ECCV 20106316 , pp.210-223,2010.

[24] R. Salakhutdinov , G. Hinton."Semantic hashing.Int. J. Approximate Reasoning,"vol. 50,pp. 969-978,2009.

[25] Ince, Turker, Serkan Kiranyaz, LeventEren, Murat Askar, and MoncefGabbouj."Real-time motor fault detection by 1-D convolutional neural networks".IEEE Transactions on Industrial Electronics,vol.63,no.11, pp.7067-7075. (2016)

[26] Tiwari, Rohit, K .Vijay Gupta ,and P.K Kankar." Bearing fault diagnosis based on multi-scale permutation entropy and adaptive neuro fuzzy classifier".Journal of Vibration and Control, vol.21,no.3, pp.461-467,2015.
[27] Kang, Myeongsu, Jaeyoung Kim, Jong-Myon Kim, Andy Tan CC, Eric Kim Y, and Byeong-Keun Choi."Reliable fault diagnosis for low-speed bearings using individually trained support vector machines with kernel discriminative feature analysis".IEEE Transactions on Power Electronics, vol.30,no.5, pp.2786-2797,2015.

[28] Kang, Myeongsu, Jaeyoung Kim, and Jong-Myon Kim. "Reliable fault diagnosis for incipient low-speed bearings using fault feature analysis based on a binary bat algorithm".Information Sciences, vol.294, pp.423-438. 2015.

[29] Li, Shaobo, Guokai Liu, Xianghong Tang,JianguangLu, and Jianjun Hu." An Ensemble Deep Convolutional Neural Network Model with Improved DS Evidence Fusion for Bearing Fault Diagnosis.Sensors,"vol. 17,no.8, pp.1729,2017

\section{Creative Commons Attribution License 4.0 (Attribution 4.0 International, CC BY 4.0)}

This article is published under the terms of the Creative Commons Attribution License 4.0 https://creativecommons.org/licenses/by/4.0/deed.en_US 